\title{
Resection of von Hippel Lindau Related Brainstem Hemangioblastoma
}

\author{
${ }^{1}$ Surgical Neurology Branch, National Institute for Neurological \\ Diseases and Stroke, Bethesda, Maryland, United States \\ 2 Neuro-Oncology Branch, National Cancer Institute, Bethesda, \\ Maryland, United States \\ ${ }^{3}$ Neurosurgery Unit for Pituitary and Inheritable Diseases, National \\ Institute for Neurological Diseases and Stroke, Bethesda, Maryland, \\ United States
}

Dominic Maggio $^{1}$ Jared S. Rosenblum ${ }^{1,2(1)}$ Prashant Chittiboina ${ }^{1,3(0)}$

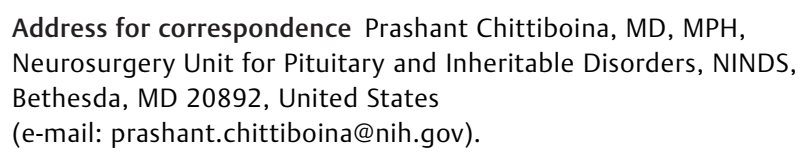
Neurosurgery Unit for Pituitary and Inheritable Disorders, NINDS, Bethesda, MD 20892, United States (e-mail: prashant.chittiboina@nih.gov).

J Neurol Surg B 2019;80(suppl S4):S348.

\section{Abstract \\ Keywords \\ - von Hippel Lindau \\ - hemangioblastoma \\ - brainstem \\ - syrinx}

Patients with von Hippel Lindau (VHL) disease develop multiple central nervous system hemangioblastomas (HB). The surgical resection of VHL-HBs is the standard of clinical care when these HBs become symptomatic due to tumor growth, edema, or cyst formation. VHL-HBs frequently present at the obex of the brainstem, making this a challenging surgical problem. Here, we present a case of a large symptomatic brainstem VHL-HB that was resected using a dorsal midline approach and midline myelotomy. This 35-year-old man with VHL disease and multiple prior resections of cerebellar hemangioblastomas presented with progressive bilateral upper extremity weakness, ataxia, and dysphagia. The accompanying two-dimensional (2D) video demonstrates the key techniques for resection of this brainstem hemangioblastoma, including recognizing the pial presentation, sharp pial/subpial dissection, and en bloc removal. Subsequently, we discuss circumferential dissection, identification, and division of feeding vessels. We also demonstrate key maneuvers to recognize and divide the main arterial pedicle while preserving the anterior spinal artery. The patient tolerated the resection without complication and the patient was discharged home after 6 days at his baseline neurologic function. This work was supported by the Intramural Research Program of the National Institutes of Health. The link to the video can be found at: https://youtu.be/mb2VqcLpxDQ.
Conflict of Interest

None declared.

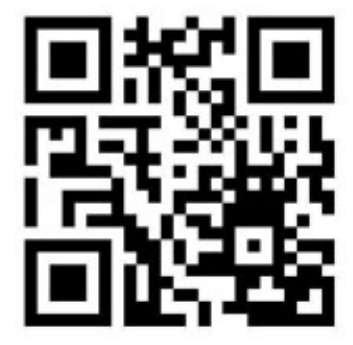

received

April 1, 2019

accepted

August 25, 2019

published online

October 22, 2019

www.thieme.com/skullbasevideos

www.thieme.com/jnlsbvideos

DOI https://doi.org/

10.1055/s-0039-1700511. ISSN 2193-6331. (c) 2019 Georg Thieme Verlag KG Stuttgart · New York
License terms

(c) (i) $\ominus$ (5) 